\title{
Coherent Resonances Observed in the Dissociative Electron Attachments to Carbon Monoxide
}

\author{
Xu-Dong Wang†, Chuan-Jin Xuan†, Yi Luoł‡, and Shan Xi Tiant* \\ $\dagger$ Hefei National Laboratory for Physical Sciences at the Microscale, \\ Center of Advanced Chemical Physics and Department of Chemical Physics, \\ University of Science and Technology of China, Hefei, Anhui 230026, China and \\ $\ddagger$ Department of Theoretical Chemistry and Biology, School of Biotechnology, \\ Royal Institute of Technology, S-106 91, Stockholm, Sweden
}

\begin{abstract}
Succeeding our previous finding about coherent interference of the resonant states of $\mathrm{CO}^{-}$formed by the low-energy electron attachment [Phys. Rev. A 88, 012708 (2013)], here we provide more evidences of the coherent interference, in particular, we find the state configuration change in the interference with the increase of electron attachment energy by measuring the completely backward distributions of the $\mathrm{O}^{-}$fragment ion of the temporary $\mathrm{CO}^{-}$in an energy range $11.3-12.6 \mathrm{eV}$. Therefore, different pure states, namely, coherent resonances, can be formed when the close-lying resonant states are coherently superposed by a broad-band electron pulse.

PACS numbers: $34.80 . \mathrm{Ht}$
\end{abstract}


Dissociative electron attachment (DEA) is a novel electron inelastic scattering process, in which the temporary negative ion is a resonant system $\left(e^{-}-\mathrm{M}\right)$ for an atomic or molecular target $\mathrm{M}$ [1]. This temporary anion $\mathrm{M}^{-}$will undergo dissociation or electron auto-detachment [2]. By measuring the momentum distributions of the negatively charged fragment, one can have insights into the formation and dissociation dynamics of $\mathrm{M}^{-}\left[\begin{array}{ll}3 & 6\end{array}\right]$. Different resonant states of $\mathrm{M}^{-}$can be formed in the low-energy electron attachments to $\mathrm{M}$, and locate energetically lower or higher than the eigenstates of the neutral $\mathrm{M}$, due to the coupling between the the eigenstate and the continuum background of the excess $e^{-}$[2, 7]. Obviously, the resonant states easily interact or couple with each other by the aid of the continuum background when these states are energetically close [8]. In our recent study of the DEA to carbon monoxide molecule, a coherent resonance as the result of quantum interference among ${ }^{2} \Pi,{ }^{2} \Delta$, and ${ }^{2} \Phi$ resonant states of $\mathrm{CO}^{-}$was found at $10.6 \mathrm{eV}$ and responsible for the completely backward distribution of the fast $\mathrm{O}^{-}$fragment ion [6]. The fast $\mathrm{O}^{-}$ion is produced via $e^{-}+\mathrm{CO} \rightarrow$ $\mathrm{C}\left({ }^{3} \mathrm{P}\right)+\mathrm{O}^{-}$(Process I), while the much slow one (with the kinetic energy near zero $\mathrm{eV}$, the left panel of FIG. 2c in ref.[6]) should be the yield of Process II $\left[e^{-}+\mathrm{CO} \rightarrow \mathrm{C}\left({ }^{1} \mathrm{D}\right)+\mathrm{O}^{-}\right]$ [4]. With the increase of the attachment energy, the slow $\mathrm{O}^{-}$ion becomes fast by sharing the excess energy with the metastable $\mathrm{C}\left({ }^{1} \mathrm{D}\right)$. It is unknown whether the same or similar quantum interference influences the momentum distributions of the $\mathrm{O}^{-}$ion from Process II.

In this communication, we report that the coherent resonance continuously leads to the rainbow-like momentum distributions of the $\mathrm{O}^{-}$ion produced via Process II, namely, the backward angular distribution with respect to the electron incident direction, by using anion velocity time-sliced map imaging technique[6, 9]. More interestingly, with the increase of attachment energy, the coherent interference is changed from the configuration of ${ }^{2} \Pi,{ }^{2} \Delta$, and ${ }^{2} \Phi$ resonant states to that of ${ }^{2} \Sigma,{ }^{2} \Delta$, and ${ }^{2} \Phi$ resonant states, implying the existences of two different coherent resonances. A new dissociation channel, $e^{-}+\mathrm{CO} \rightarrow \mathrm{C}\left({ }^{1} \mathrm{~S}\right)+\mathrm{O}^{-}$ (Process III), is found in the measurement at $12.3 \mathrm{eV}$, which was not reported before [4].

The experiments were done with our home-made anion momentum imaging apparatus [6, 9]. In brief, an effusive molecular beam of $\mathrm{CO}$ (along $y$ axis) was perpendicular to the pulsed electron beam (along $x$ axis, the thermal energy spread of $0.5 \mathrm{eV}$ ) which was collimated with the homogeneous magnetic field produced by a pair of Helmholtz coils. The $\mathrm{O}^{-}$ions were periodically pushed out of the reaction area (volume size was less than $2 \times 2 \times 2$ $\mathrm{mm}^{3}$ ) and expanded to form a Newton sphere which passed through a series of the circle 
electrodes and time-of-flight tube (along $y$ axis). The high momentum resolution $(\triangle v / v \leq$ $2 \%$ ) was achieved by these well-designed electrodes [9]. The $\mathrm{O}^{-}$ions were detected with a set of triple microchannel plates and a phosphor screen (its diameter is $40 \mathrm{~mm}$ ). The central time-sliced image of $\mathrm{O}^{-}$was recorded by the application of a narrow time-gate pulse voltage (width $45 \mathrm{~ns}$ ) on the last microchanel plate and with a CCD camera. In our previous experiments [6], the Newton sphere of the $\mathrm{O}^{-}$ions produced via Process II was too small to be sliced. According to the working principle of the ion velocity slice imaging technique, this Newton sphere was magnified by reducing the voltages on the pusher and the other electrodes in a proper ratio [10]. The kinetic energy of the ion fragment was obtained after calibration with the experimental data available in the literature.

As shown in FIG. 1(a-e), the $\mathrm{O}^{-}$momentum images for Process II appear to grow in size radially with the increase of the electron attachment energy; while the most of the ions produced in Process I are lost (FIG. 1a and 1b) because the Newton sphere of the latter is too large and out of the detector. At the electron attachment energies of 12.4 and 12.6 $\mathrm{eV}$, the much slower $\mathrm{O}^{-}$ions appear as the central points of the images in FIG. 1(d) and 1(e). These slower ions are produced in Process III, because the threshold energy of this process is $12.30 \mathrm{eV}$ [11]. The kinetic energies of the $\mathrm{O}^{-}$ions via Process II are plotted against the electron attachment energy in FIG. 1(f). The fitted line intersects the $x$ axis at $10.9 \mathrm{eV}$, in excellent agreement with the calculated threshold $10.88 \mathrm{eV}$ of Process II [11]. Our present and previous [12] studies show the high experimental accuracy in determining the dissociation thresholds. More importantly, the angular distributions of the $\mathrm{O}^{-}$ions produced in both Processes II and III clearly indicate the backward scattering character, which is quite similar to that observed in Process I [6].

As discussed in our previous study for Process I, the coherent interference of ${ }^{2} \Pi,{ }^{2} \Delta$, and ${ }^{2} \Phi$ resonant states was proposed to interpret the completely backward distribution of $\mathrm{O}^{-}$at $10.6 \mathrm{eV}$ [6]. Is this mechanism still applicable for the present observations? The $\mathrm{O}^{-}$angular distributions of Process II are plotted in FIG. 2 where the ion intensities are obtained by integrations of the ion signals within the selected kinetic energy ranges. On the basis of the theoretical model for the fragment momentum distribution in the DEA to diatomic molecule [13], the differential cross section $\sigma_{D E A}$ is,

$$
\sigma_{D E A} \propto \sum_{|\mu|}\left|\sum_{j=1, l=|\mu|} c_{j} i e^{i \delta_{j}} Y_{l \mu}(\theta, \zeta)\right|^{2}
$$


where the incident electron is described with a series of partial waves with different angular momenta $l, c_{j}$ is the weighing parameters of each wave $j, \delta_{j}$ is the phase lag among the partial waves due to the interaction between the incoming electron and target, and $Y_{l \mu}$ is the spherical harmonics. The parameter $\mu$ has a absolute value of $\left|\Lambda_{f}-\Lambda_{i}\right|$, representing the difference in the projection of the angular momenta along the internuclear axis for the neutral $(i)$ and anionic resonant $(f)$ states. In the simple cases, one or several resonant states (mixed state) should be considered, corresponding to one or serval $|\mu|$ values. If two or more resonant states $\varphi_{i}$ as a superposition state, $\varphi=\sum \varphi_{i}, i=1,2, \ldots$, contribute to the cross section, the quantum interference among these resonant states $\varphi_{i}$ is introduced in a straightforward way $[\underline{6}]$,

$$
\sigma_{D E A} \propto \sum_{\alpha, \beta} I_{\alpha, \beta}+2 \sum_{\alpha \neq \beta} \sqrt{I_{\alpha} I_{\beta}} \cos \phi_{\alpha \beta}
$$

where $I_{\alpha}$ is the amplitude contribution in the form of $\left|\sum c_{j} i e^{i \delta_{j}} Y_{l \mu}\right|^{2}$ for resonant state $\alpha$ and $\phi_{\alpha \beta}$ is the phase shift or difference between $\alpha$ and $\beta$ states. Following the procedure proposed in our previous work [6], one or several states (eq.1) and then the interference form (eq.2) were used in fitting the experimental angular distributions, indicating the best fitting results by using eq.2 (the solid and dashed curves in FIG. 2).

The solid curves represent the data fitting by considering of the quantum interference of ${ }^{2} \Pi,{ }^{2} \Delta$, and ${ }^{2} \Phi$ resonant states, where two partial waves are used in each $I$ term: $l=1(p)$ and $2(d)$ for ${ }^{2} \Pi, l=2(d)$ and $3(f)$ for ${ }^{2} \Delta$, and $l=3(f)$ and $4(g)$ for ${ }^{2} \Phi[6]$. At the energies higher than $11.8 \mathrm{eV}$, two configurations of the state interference, ${ }^{2} \Pi+{ }^{2} \Delta+{ }^{2} \Phi$ and ${ }^{2} \Sigma+{ }^{2} \Delta+{ }^{2} \Phi$, are used, where ${ }^{2} \Sigma$ is the fifth resonant state of $\mathrm{CO}^{-}$and all of them are close-lying in the Franck-Condon region of the electron vertical attachment [14]. The interference of ${ }^{2} \Pi$, ${ }^{2} \Delta$, and ${ }^{2} \Phi$ resonant states can give the good fittings at the lower energies 11.3 and 11.8 $\mathrm{eV}$, while some bumps appear and the fitting curves clearly deviate from the experimental data in the forward direction at the higher energies 12.4 and $12.6 \mathrm{eV}$. The interference of ${ }^{2} \Sigma,{ }^{2} \Delta$, and ${ }^{2} \Phi$ resonant states can describe no distributions in the forward direction (see the dashed curves in FIG. 2) at these higher energies. At $12.1 \mathrm{eV}$, we cannot identify the best one from these two interference modes. In Table I, the fitting parameters are listed. We notice that the sum of phase shifts among the resonant states is consistently satisfied with $\phi_{12}+\phi_{23}+\phi_{31} \approx 2 \pi$ rad. The phase sum of $2 \pi$ evidences the coherency of quantum interference or the existence of coherent resonance; moreover, two coherence resonances are 
observed in this work.

To reveal the different contributions of all terms in eq.2, the angular distribution from each term is plotted in FIG. 3 by using the fitting parameters. In FIG. 3a, the sum of all terms is the fitting curve at $11.3 \mathrm{eV}$ for the interference of ${ }^{2} \Pi,{ }^{2} \Delta$, and ${ }^{2} \Phi$ states. The ${ }^{2} \Phi$ state component shows the maximum around $80^{\circ}$, two maxima appear at $60^{\circ}$ and $120^{\circ}$ for ${ }^{2} \Delta$ state, while one maximum around $130^{\circ}$ arises from ${ }^{2} \Pi$ state. The forward distributions from $\Delta, \Phi$, and the cross term $\Pi * \Delta$ are counteracted with the cross terms $\Delta * \Phi$ and $\Pi * \Phi$. In FIG. 3b, the sum curve corresponds to the fitting result at $12.6 \mathrm{eV}$ for the interference of ${ }^{2} \Sigma,{ }^{2} \Delta$, and ${ }^{2} \Phi$ states. The ${ }^{2} \Sigma$ state typically shows both the forward and backward distributions, and the forward ones are overall canceled with the cross terms $\Sigma * \Phi$ and $\Delta * \Phi$. In general, the cross terms, i.e., the interference terms $I_{\alpha} I_{\beta} \cos \phi_{\alpha \beta}$, play the essential roles in the completely backward distributions of the $\mathrm{O}^{-}$ions.

Although the statistic errors of the ion signal are large for Process III, the angular distributions at $12.6 \mathrm{eV}$ in FIG. 4 also indicate the backward scattering character. The peak positions of the ion intensities show some differences when the $\mathrm{O}^{-}$ions are selected in the different kinetic energy ranges. Considering the uncertain weighings of the ions with the lower kinetic energies, we only fit the angular distribution of the ions within the higher kinetic energy range of $0.15-0.20 \mathrm{eV}$ by using eq. 2 with the interference of ${ }^{2} \Sigma,{ }^{2} \Delta,{ }^{2} \Phi$ states, indicating a good fitting (dashed) curve in FIG. 4. As listed in Table I, the phase sum $\phi_{12}+\phi_{23}+\phi_{31}$ approximately equals $2 \pi$ rad again, implying that the corresponding coherence resonance should also be responsible for the backward distributions of the $\mathrm{O}^{-}$ion produced via Process III.

In summary, we demonstrate that the coherent interference of ${ }^{2} \Pi,{ }^{2} \Delta$, and ${ }^{2} \Phi$ resonant states of $\mathrm{CO}^{-}$can be established in a wide energy range, but the state configuration of the interference is changed to ${ }^{2} \Sigma,{ }^{2} \Delta$, and ${ }^{2} \Phi$ resonant states when the electron attachment energy is higher than $12.1 \mathrm{eV}$. All of the sums of phase shifts obtained in the experimental data fittings are equal to $2 \pi \mathrm{rad}$, indicating that two coherent resonances exist as the superposition sates, $\varphi_{1}={ }^{2} \Pi+{ }^{2} \Delta+{ }^{2} \Phi$ and $\varphi_{2}={ }^{2} \Sigma+{ }^{2} \Delta+{ }^{2} \Phi$. We believe that the formation of the coherent resonance is similar to the production of coherent wave packet using a broad-band ultrashort laser pulse in coherent control [15], but it is a new concept in quantum scattering and deserves the further theoretical explorations.

This work is supported by the NSFC (Grant No. 21273213) and MOST (Grant No. 
2013CB834602).

* Electronic address: sxtian@ustc.edu.cn

[1] J. R. Taylor, Scattering Theory: The Quantum Theory of Norelativistic Collisions, (John Wiely \& Sons, New York, 1972).

[2] Electron-Molecule Interactions and Their Applications, edited by L. G. Christophorou (Academic Press, New York, 1984).

[3] B. Boudaïfa, P. Cloutier, D. Hunting, M. A. Huels, and L. Sanche, Science 287, 1658 (2000).

[4] R. I. Hall, I. Čadež, C. Schermann, and M. Tronc, Phys. Rev. A 15, 599 (1977).

[5] C. König, J. Kopyra, I. Bald, and E. Illenberger, Phys. Rev. Lett. 97, 018105 (2006).

[6] S. X. Tian, B. Wu, L. Xia, Y.-F. Wang, H.-K. Li, X.-J. Zeng, Y. Luo, and J. Yang, Phys. Rev. A 88, 012708 (2013).

[7] Y.-F. Wang and S. X. Tian, Phys. Rev. A 85, 052709 (2012).

[8] E. Narevicius and N. Moiseyev, Phys. Rev. Lett. 84, 1681 (2000).

[9] B. Wu, L. Xia, H.-K. Li, X.-J. Zeng, and S. X. Tian, Rev. Sci. Instrum. 83, 013108 (2012).

[10] H.-K. Li, L. Xia, Z.-J. Zeng, and S. X. Tian, J. Phys. Chem. A 117, 3176 (2013).

[11] The threshold of Process I is $9.62 \mathrm{eV}$ [4], and $\mathrm{C}\left({ }^{1} \mathrm{D}\right)$ and $\mathrm{C}\left({ }^{1} \mathrm{~S}\right)$ are 1.26 and $2.68 \mathrm{eV}$ higher than $\mathrm{C}\left({ }^{3} \mathrm{P}\right)$, respectively [V. Kaufman and J. F. Ward, J. Opt. Soc. Am. 56, 1591 (1966)].

[12] L. Xia, X.-D. Wang, C.-J. Xuan, X.-J. Zeng, H.-K. Li, S. X. Tian, Y. Pan, and K.-C. Lau, J. Chem. Phys. 140, 041106 (2014).

[13] T. F. O'Malley, Phys. Rev. 150, 14 (1966); T. F. O'Malley and H. S. Taylor, ibid. 176, 207 (1968).

[14] L. A. Morgan and J. Tennyson, J. Phys. B 26, 2429 (1993).

[15] K. Ohmori, Annu. Rev. Phys. Chem. 60, 487 (2009). 
Table I. Fitting parameters obtained by using eq.2 for the angular distributions of $\mathrm{O}^{-}$ produced via Processes II and III.

\begin{tabular}{llll}
\hline${ }^{2} \Pi+^{2} \Delta+{ }^{2} \Phi$ & & \\
\hline Attachment Energy (eV) & $11.3(\mathrm{P}$ II) & 11.8 (P II) & 12.1 (P II) \\
Weighing ratio & & & \\
$c_{1}: c_{2}: c_{3}:$ & $0.98: 0.52: 1.00: 0.90: 0.46: 0.37: 0.68: 0.49: 1.00:$ \\
$c_{4}: c_{5}: c_{6}$ & $0.99: 1.67: 0.31$ & $0.68: 1.00: 0.62$ & $0.46: 0.52: 0.25$
\end{tabular}

Phase lags (rad)

$\begin{array}{llll}\left({ }^{2} \Pi\right) \delta_{d}-\delta_{p} & 1.57 & 1.57 & 0.40 \\ \left({ }^{2} \Delta\right) \delta_{f}-\delta_{d} & 3.05 & -1.36 & -1.57 \\ \left({ }^{2} \Phi\right) \delta_{g}-\delta_{f} & -1.56 & -1.77 & -2.28\end{array}$

Phase shift (rad)

\begin{tabular}{llll}
$\phi_{12}\left({ }^{2} \Pi-{ }^{2} \Delta\right)$ & 0.00 & 0.16 & 3.10 \\
$\phi_{23}\left({ }^{2} \Delta-{ }^{2} \Pi\right)$ & 3.01 & 3.20 & 0.00 \\
$\phi_{31}\left({ }^{2} \Phi-{ }^{2} \Pi\right)$ & 3.07 & 3.15 & 3.16 \\
\hline
\end{tabular}

${ }^{2} \Sigma+^{2} \Delta+^{2} \Phi$

Attachment Energy (eV) 12.4 (P II) 12.6 (P II) 12.6 (P III)

Weighing ratio
$c_{1}: c_{2}: c_{3}:$
0.05:0.12:1.00: 0.10:0.44:1.00: 0.11:0.19:1.00:
$c_{4}: c_{5}: c_{6}$
0.41:0.80:0.00 $\quad 0.78: 0.67: 0.32 \quad 0.26: 0.97: 0.04$

Phase lags (rad)

\begin{tabular}{llll}
$\left({ }^{2} \Sigma\right) \delta_{p}-\delta_{s}$ & 0.40 & 0.21 & 1.91 \\
$\left({ }^{2} \Delta\right) \delta_{f}-\delta_{d}$ & 2.35 & 0.04 & 1.04 \\
$\left({ }^{2} \Phi\right) \delta_{g}-\delta_{f}$ & -3.14 & -1.57 & 1.69 \\
Phase shift (rad) & & & \\
$\phi_{12}\left({ }^{2} \Sigma-{ }^{2} \Delta\right)$ & 1.84 & 1.43 & 1.53 \\
$\phi_{23}\left({ }^{2} \Delta-{ }^{2} \Sigma\right)$ & 1.19 & 2.33 & 1.39 \\
$\phi_{31}\left({ }^{2} \Phi-{ }^{2} \Sigma\right)$ & 3.29 & 2.46 & 3.14 \\
\hline
\end{tabular}



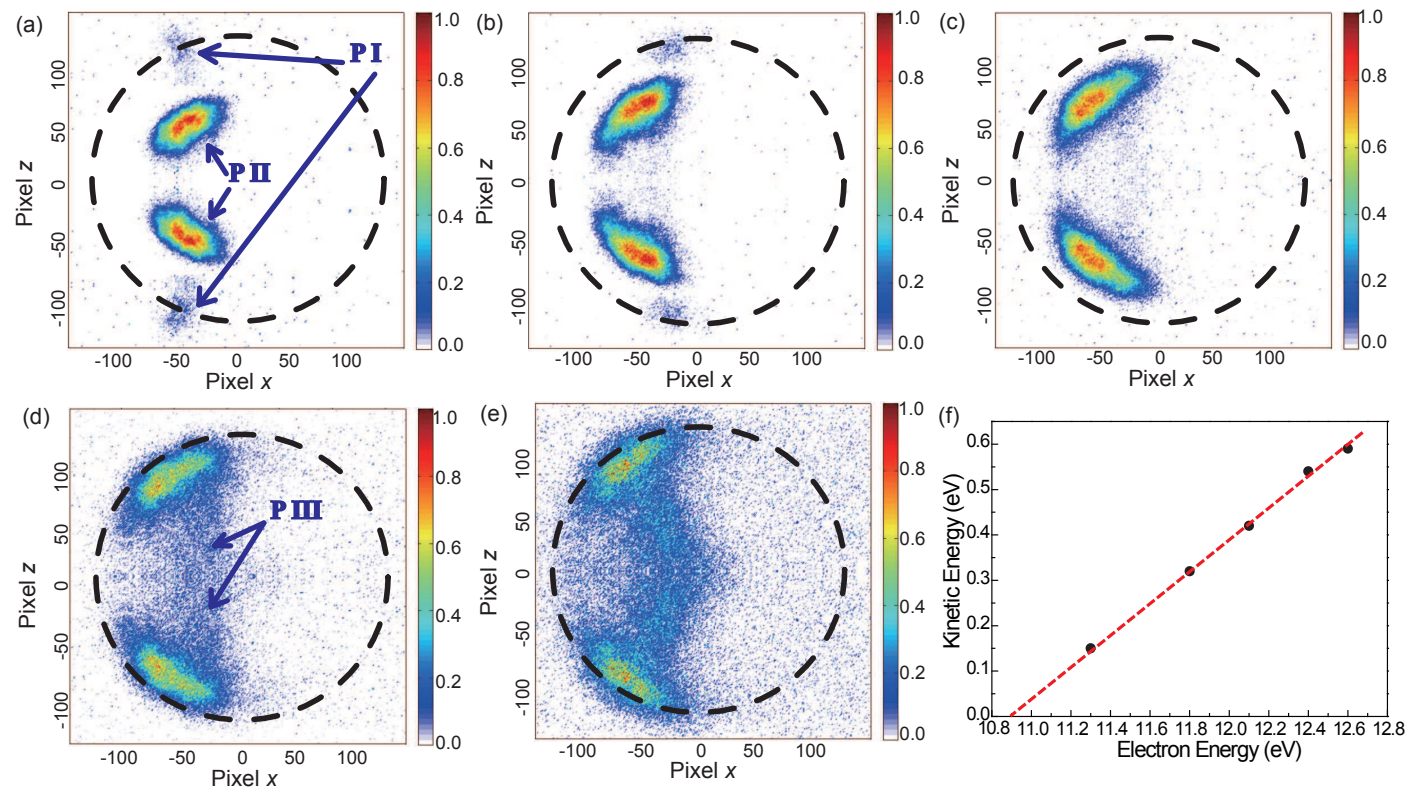

FIG. 1: Sliced images of $\mathrm{O}^{-}$recorded at 11.3 (a), 11.8 (b), 12.1 (c), 12.4(d), and 12.6 $\mathrm{eV}(\mathrm{e})$, where the electron incident direction is from left to right and through the image center, the ion intensity is normalized respectively, P I, P II, P III denote the different dissociation processes (see the details in text), and the broken circle represents the effective area (diameter $40 \mathrm{mmm}$ ) of the detector. (f) The most probable kinetic energy of $\mathrm{O}^{-}$ (produced via P II) in terms of the electron attachment energy. 


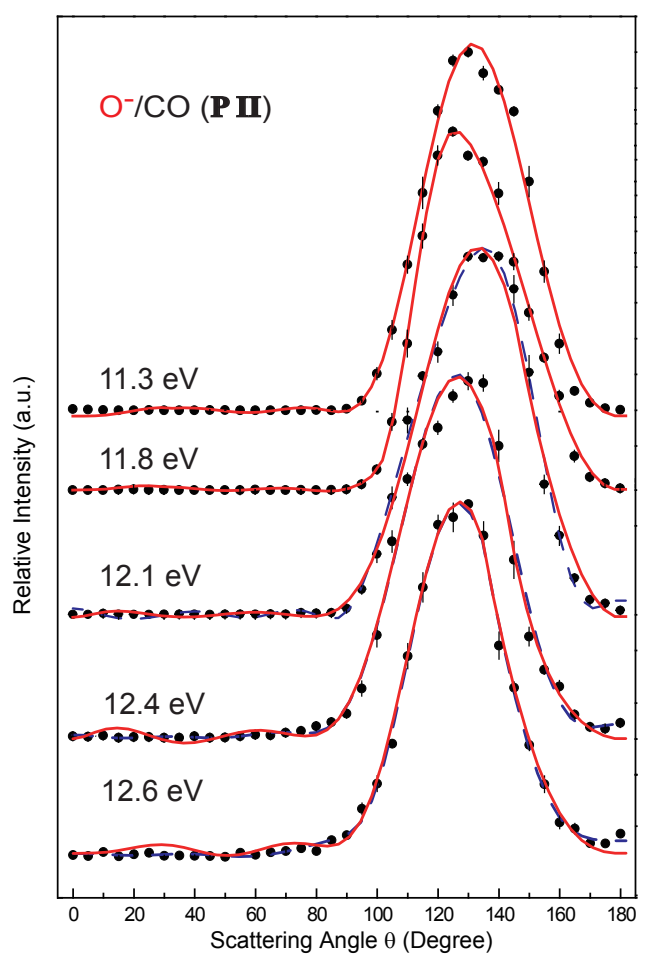

FIG. 2 Angular distribution of $\mathrm{O}^{-}$ions from Process II: solid circles, the experimental data (the selected kinetic energy range: $0.14-0.24 \mathrm{eV}$ at $11.3 \mathrm{eV}, 0.25-0.35 \mathrm{eV}$ at $11.8 \mathrm{eV}$, $0.36-0.46 \mathrm{eV}$ at $12.1 \mathrm{eV}, 0.49-0.59 \mathrm{eV}$ at $12.4 \mathrm{eV}, 0.54-0.64 \mathrm{eV}$ at $12.6 \mathrm{eV})$; solid curves, the data fitting with the quantum interference of ${ }^{2} \Pi,{ }^{2} \Delta$, and ${ }^{2} \Phi$; dashed curves, the data fitting with the quantum interference of ${ }^{2} \Sigma,{ }^{2} \Delta$, and ${ }^{2} \Phi$. 


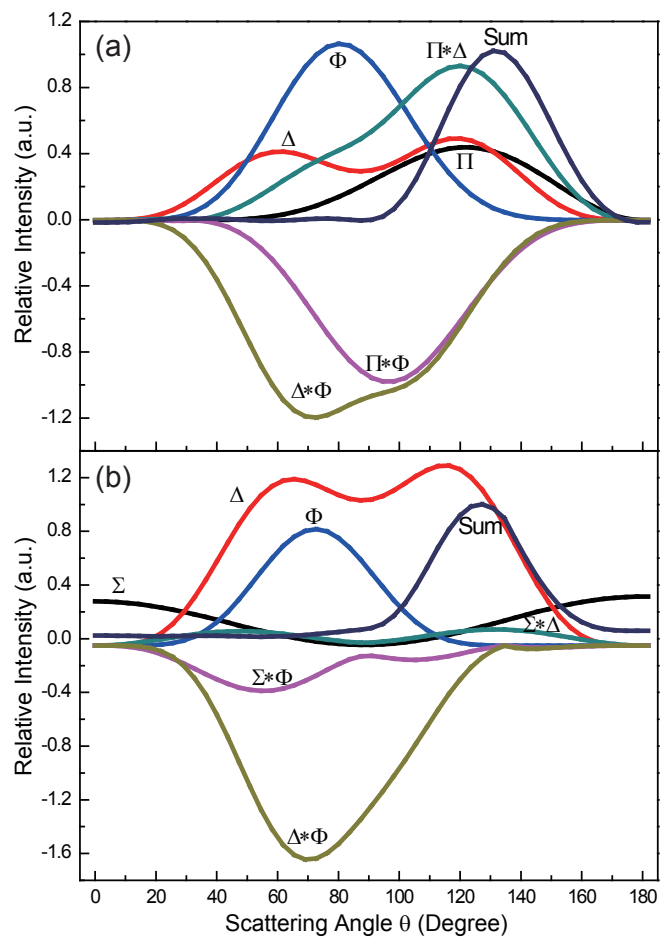

FIG. 3 Contributions of each term in eq.2 to the angular distribution using the fitting parameters for the quantum interference of ${ }^{2} \Pi,{ }^{2} \Delta$, and ${ }^{2} \Phi$ at $11.3 \mathrm{eV}$ (a) and that of ${ }^{2} \Sigma$, ${ }^{2} \Delta$, and ${ }^{2} \Phi$ at $12.6 \mathrm{eV}(\mathrm{b})$. 


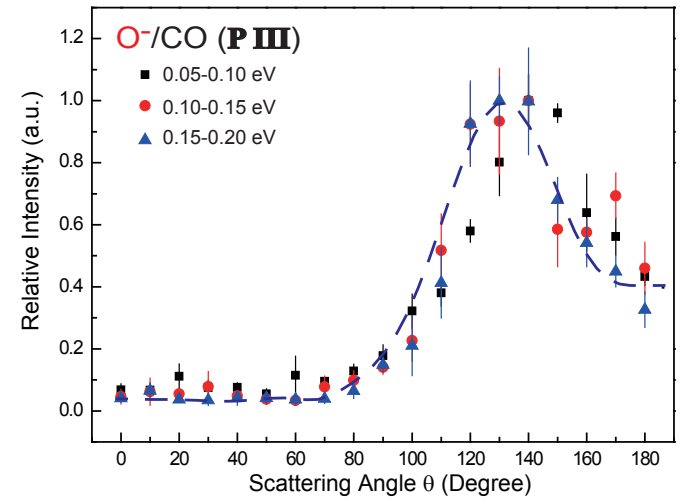

FIG. 4 Angular distribution of $\mathrm{O}^{-}$ions from Process III at $12.6 \mathrm{eV}$ where the dashed curve represents the data fitting with the quantum interference of ${ }^{2} \Sigma,{ }^{2} \Delta$, and ${ }^{2} \Phi$ for the ions with the kinetic energy of $0.15-0.20 \mathrm{eV}$. 